\title{
Spatio-temporal investigations on the triggering of pellet induced ELMs
}

\author{
G. Kocsis ${ }^{1}$, S. Kálvin ${ }^{1}$, P.T. Lang ${ }^{2}$, M. Maraschek ${ }^{2}$, J. \\ Neuhauser $^{2}$, W. Schneider ${ }^{2}$, T.Szepesi ${ }^{1}$ and ASDEX Upgrade \\ Team $^{2}$ \\ ${ }^{1}$ KFKI Research Institute for particle and Nuclear Physics, EURATOM Association, \\ P.O. Box 49, H-1525 Budapest, Hungary \\ ${ }^{2}$ Max-Planck-Institut für Plasmaphysik, EURATOM Association, Boltzmannstr. 2, \\ 85748 Garching, Germany
}

E-mail: kocsis@rmki.kfki.hu

\begin{abstract}
.
Pellets injected into type-I ELMy H-mode discharges are known to trigger ELMs. In order to understand the underlying processes the triggering mechanism was investigated in this paper. The major questions of the investigations to be answered were: at which magnetic surface was the ELM initiated and what was the corresponding perturbation caused by the ablating pellet. During the investigations the natural ELM cycle was probed by injecting pellets from the HFS of ASDEX Upgrade tokamak with significantly lower frequency than the natural ELM frequency. To determine the location of the seed perturbation of the ablating pellet triggering an ELM, the dynamics of the triggered ELMs was linked to the time history of the pellet position in the plasma. The ELM onset was determined by analyzing magnetic pick-up coil signals and its delay relative to the time when the pellet crossed the separatrix was measured as a function of the pellet velocity. Supposing that to trigger an ELM a pellet has to reach a certain magnetic surface of the plasma independently of its mass and velocity, the most probable location of the seed perturbation was found to be at the middle of the pedestal - at the high plasma pressure gradient region. The onset of the MHD signature of the ELMs was detected about $50 \mu s$ after the pellet reached the seed position. According to our observations ELMs can be triggered either by the cooling of the pedestal region causing sudden increase of the pedestal plasma pressure gradient driving the plasma to the unstable region of the ballooning mode or by the strong MHD perturbation triggering an instability developing into an ELM.
\end{abstract}

PACS numbers: 52.55.Fa, 52.40.Hf, 52.35.Py 


\section{Introduction}

The type-I ELMy H-mode is foreseen to be a basic operation mode for ITER, the hitherto largest tokamak experiment coming up in the next decade [1]. However, this mode is accompanied by the presence of edge-localized modes (ELMs), MHD-instabilities periodically breaking the transport barrier, which result in a sudden expulsion of plasma particles and energy on a (sub-)millisecond timescale [2]. The expelled energy ( $\left.d W_{E L M}\right)$ is deposited on first wall components, resulting in a high transient heat load on divertor plates which could reduce component lifetimes to an unacceptably low level. It was found empirically in several machines, that ELMs transport roughly $20 \%$ of the heating power out of the confined plasma independently of the ELM frequency therefore the energy expelled during a single ELM is inversely proportional with the ELM frequency $\left(f_{E L M}\right)[3]$. As can be seen, ELM size $\left(d W_{E L M}\right)$ increases significantly with machine size, and scalings derived from existing large tokamaks indicate that the power load imposed by naturally occurring ELMs on the ITER divertor components may exceed tolerable values by a factor of 5-10 [4]. On the other hand ELM frequency is also a key parameter for the impurity control and for the avoidance of radiative instabilities in H-mode [5]. All of these considerations indicate that to develop a technique for ELM frequency control is highly desired.

Pellets, originally designed for refueling, are known to trigger type-I ELMs, if injected into an ELMy H-mode discharge [6, 7, 8, 9]. This phenomenon provides the possibility of ELM frequency - and hence the divertor power load and impurity content - control (pacemaking) by continuous pellet injection. This approach was demonstrated at ASDEX Upgrade tokamak, where pellets were injected from the magnetic high field side (HFS) by an injector system originally designed for refueling [10]. The full control of the ELM activity was taken over by imposing a pellet injection frequency higher than the intrinsic $f_{E L M}$ by a factor of 1.5 or more. A statistical analysis of these scenarios revealed a significant reduction of both the average and maximum ELM size [10].

The pellet ELM pacemaking has been found to be one of the most promising techniques to mitigate ELM caused power load. Pellet pacing systems are under development for DIII-D [11] and JET [12] and in the concept for ITER [13] as well. The technique itself works but the underlying physical processes of the ELM triggering are not well understood although the understanding of the trigger mechanism is indispensable to extrapolate for the future experiments. Therefore our aim is to study how and where a pellet triggers an ELM.

Preliminary analysis [14] that uses simplifying assumptions has indicated that ELMs seem to be triggered once the pellet has reached a certain position in the pedestal roughly independently of the pellet parameters. Sophisticated investigations such as statistically large enough number of evaluated events, improved evaluation, statistical analysis, rigorous error estimation would be of high relevance for theoretical understanding and model validation. This will be described in this paper. The major questions of the investigations to be answered were: at which magnetic surface was the 
ELM initiated and what was the corresponding perturbation caused by the ablating pellet. Therefore we have linked the dynamics of the triggered ELM to time history of the pellet position in the plasma. A time characterizing the onset of the ELM and its delay relative to the time when the pellet crossed the separatrix and therefore the position of the pellet at the onset of the ELM was determined. These quantities were determined for different pellet parameters (mass and velocity) and from these data the location of the seed perturbation initiating an ELM was concluded.

The paper is organized as follows. Section 2 covers the experimental details: how the pellets were localized and how the ELM onset was determined. Section 3 shows the results: the dependence of the delay between the ELM onset and the time when the pellet crossed the separatrix on the pellet velocity and the variation of the ELM size on the pellet mass. The paper ends with conclusions and outlook.

\section{Experiment}

The experiments - described in this paper - have been performed on the ASDEX Upgrade tokamak injecting pellets from the high field side of the torus [15] into the type-I ELM regime of an H-mode discharge. A lower single null configuration was used with $\mathrm{I}_{P}=1 M A, \mathrm{~B}_{t}=-2.7 T, \mathrm{q}_{95}=4.9, \kappa=1.6, \delta^{u}=0.12$ and $\delta^{l}=0.37$. Stable and robust operation in the type-I ELM regime with low natural ELM frequency $\mathrm{f}_{E L M} \sim 25-45 \mathrm{~Hz}$ (depending on the actual wall conditions) was achieved by keeping the auxiliary heating power just above the L-H transition power threshold. Typically $5 \mathrm{MW}$ neutral beam injection power was applied. Impurity accumulation and density profile peaking was prevented by modest (0.8-1.3 MW) central deposited ion cyclotron resonance heating. The pedestal parameters (electron density and temperature) were similar for all discharges used in these investigations.

For the sake of ELM triggering pellets were injected into the plasma from the torus HFS. In order to avoid the disturbance of the natural ELM cycle and significant parasitic plasma fueling, perturbative ELM triggering with driving frequency $(6 \mathrm{~Hz})$ small compared to the natural ELM frequency was used. However, using this method a new parameter was introduced: trigger events occur at different times in the natural ELM cycle that is ELMs are randomly triggered at different times elapsed after the previous natural ELM (henceforward $d t_{\text {elapsed }}$ ) therefore the analysis was extended to investigate how the measured quantities depend on this elapsed time. Obviously the maximum of this elapsed time is determined by the natural ELM frequency. Since the lifetime of an ELM is in the order of a millisecond triggered ELMs for $d t_{\text {elapsed }}$ of a few millisecond could not be completely separated from the previous natural one.

Typical time traces (figure 1) show the chronology of the events during pellet ELM triggering in ASDEX Upgrade. First the pellet penetrates into the plasma and starts to ablate that is the pellet ablation monitor signal rises. About $70 \mu s$ after the ablation onset a strong quasi periodic oscillation is seen on a typical magnetic coil signal indicating the MHD signature of an ELM. A few tens of microsecond later the 
$D_{\alpha}$ emission increases in the outer divertor and in about $150 \mu s$ in the inner divertor. This LFS-HFS delay in the appearance of the ELM in the outer and inner divertor is consistent with a perturbation propagating along the field lines at the pedestal-top ion sound speed [16].

\subsection{Pellet injection scenarios}

In the investigations presented in this paper pellets with four different pellet velocities $(240,600,880,1000 \mathrm{~m} / \mathrm{s})$ were injected into ASDEX Upgrade plasmas from the HFS. To minimize the unwanted side effect of plasma fueling, the technically available smallest pellet size was used. Depending on the pellet velocity the originally identical pellet size (rectangular solid with edges of 1.4,1.4 and $1.5 \mathrm{~mm}$ ) was eroded in the HFS looping system [15] resulting in the following equivalent pellet radii (and deuterium content) before entering the plasma: $240 \mathrm{~m} / \mathrm{s}: 0.71 \mathrm{~mm}\left(9 \cdot 10^{19}\right), 600 \mathrm{~m} / \mathrm{s}: 0.67 \mathrm{~mm}\left(7 \cdot 10^{19}\right)$, $880 \mathrm{~m} / \mathrm{s}: \quad 0.58 \mathrm{~mm}\left(5 \cdot 10^{19}\right), 1000 \mathrm{~m} / \mathrm{s}: 0.51 \mathrm{~mm}\left(3 \cdot 10^{19}\right)$. It is worth noting that the pellet size (mass) has a natural random variation as well: sometimes small pieces break off resulting in a further reduced pellet size.

To characterize the similarity and differences of the four injection scenarios the ablation rates along the pellet path were calculated. The typical magnetic structure together with the designated pellet path and typical density and temperature profiles obtained from the combination of the different diagnostics and two discharges can be seen on figure 2 as a function of the distance along the pellet trajectory. In the calculation the above density and temperature profiles and an ablation rate formula obtained from a neutral gas shielding model of the pellet ablation [17] was used. From the results it is obvious that pellets start to ablate near to the separatrix and penetrate into the core plasma $(12-25 \mathrm{~cm})$ crossing the whole pedestal region for all four scenarios. At the beginning of the ablation (a few centimeter inside the separatrix) the ablation rate is nearly identical for all four cases.

\subsection{Pellet localization}

In order to determine the delay of a triggered ELM relative to the pellet injection, a reference time of pellet injection has to be selected. Our aim is to determine the position of the seed perturbation therefore we have to know where the pellet was at the reference time point. Accordingly, the time when the pellet crossed the separatrix is selected as reference time.

To gain detailed information about the pellet ablation, pellet trajectory and acceleration during pellet ablation in the plasma an observation system [18] was developed at ASDEX Upgrade tokamak consisting of several 2D cameras that detect the distribution of the radiation emitted by the pellet cloud and photo diodes that measure the time evolution of the light emission (ablation monitor signal, see figure 1).

In the experiments presented here a tangential view of the poloidal cross section of the pellet injection was imaged onto the CCD sensors. Cameras were triggered after a 
preselected delay once the ablation monitor signal exceeded a set threshold. For every pellet our cameras recorded 1-5 images onto the same frame using exposure time of 5-10 $\mu s$ and 95-90 $\mu s$ off time. In order to get the time of each exposure during the plasma discharge, the camera trigger signal was digitized in the ASDEX Upgrade data acquisition system. As a typical example, the ablation monitor signal and the time of the five $10 \mu \mathrm{s}$ long exposures separated by $90 \mu \mathrm{s}$ can be seen on the upper part of figure 3 . In this case the pellet lifetime is about $600 \mu \mathrm{s}$ therefore five separated patches will be visible on the according multiple exposure image. This allowed us to determine the position of the pellet cloud [18] attached to the pellet itself [19] and to match the time of every given exposure. It was supposed that the location of the pellet in its cloud is where the maximum of the radiation is, therefore for each exposure the pellet coordinates $\left(R_{i}, z_{i}\right.$ : major radius, vertical coordinate, $\left.\mathrm{i}=1,2, \ldots, 5\right)$ and the according $t_{i}$ times could be reconstructed assuming that the pellet does not leave the poloidal cross section of the injection. These positions and the time markers can be seen on the lower graph of figure 3 .

To determine the time when a pellet crossed the separatrix the pellet trajectory has to be reconstructed. Two different methods were applied and compared. The simple one (A) supposes that a pellet flies on a straight trajectory with a constant velocity from the end of the flight tube, where the pellet enters into the torus $\left(R_{0}, z_{0}\right)$ until the first exposure - that is located inside the separatrix - is detected on the camera image $\left(R_{1}, z_{1}\right)$. In this case the time of the crossing can easily be calculated by using the initial accurately known pellet velocity. For the determination of the flux surfaces/separatrix the reconstruction of magnetic equilibrium with CLISTE code [20] is used and an accuracy of $\pm 1 \mathrm{~cm}$ along the pellet path was achieved.

The more accurate trajectory reconstruction method (B) takes into account that pellets are accelerated in the radial direction to the LFS [18] therefore only its vertical velocity component is constant and the trajectory is curved as well. The trajectory can be described with reasonable accuracy by the following expressions

$$
\begin{gathered}
z=z_{0}-V_{0} \cdot t \sin (\alpha) \\
R=R_{0}+V_{0} \cdot t \cos (\alpha)+C \cdot t^{3}
\end{gathered}
$$

where $V_{0}$ is the pellet velocity before entering the torus, $\alpha$ is the injection angle and $C$ is a constant. As it is seen here, a temporally linearly increasing radial acceleration was assumed. Eliminating $t$ from eq. 2 by using eq.1, an $R=R(z)$ function is obtained. The unknown constants ( $\alpha$ and $C /\left(V_{0}^{3} \cdot \sin ^{3}(\alpha)\right)$ ) are determined by fitting this function onto the camera image maximizing the radiation integrated along the trajectory. For the example shown above the reconstructed pellet trajectory can be seen on figure 3 . Once the parameters of the trajectory are known the coordinates of its crossing with the separatrix and the according time can be calculated by fitting the linear $\mathrm{z}=\mathrm{z}(\mathrm{t})$ function onto $\left(z_{i}, t_{i}\right)$.

Using both methods it was noticed that the second one (B) could be applied only for 
$60 \%$ of the pellets because e.g. pellets which have a lifetime of 100-200 $\mu$ s could be seen only on one or two exposures therefore the fitting procedures could not be performed. The simple method (A) works for all cases therefore in the investigations detailed in the next sections this was used. The separatrix crossing times obtained by the two methods were compared for the cases where both methods could be applied and it was observed that the typical difference was less $10 \mu \mathrm{s}$. It was larger for slower pellets $\left(V_{P}=240 \mathrm{~m} / \mathrm{s}\right.$ : average value: $7 \mu \mathrm{s}$, standard deviation: $15 \mu \mathrm{s})$ and smaller for faster pellets $\left(V_{P}=600\right.$ $\mathrm{m} / \mathrm{s}$ : average value: $4 \mu s$, standard deviation: $8 \mu s$ )

Summarizing the following factors determine the uncertainty of the calculated crossing time: the inaccuracy of the determination of the separatrix on the HFS $( \pm 1 \mathrm{~cm})$, the inaccuracy of the spatial calibration of the camera images $( \pm 0.5 \mathrm{~cm})$ and the estimated inaccuracy of the used trajectory reconstruction method $\left( \pm 20 \mu \mathrm{s}\right.$ for $V_{P}=240$ $\mathrm{m} / \mathrm{s}$ and $\pm 10 \mu s$ for $\left.V_{P} \geq 600 \mathrm{~m} / \mathrm{s}\right)$.

\subsection{ELM onset determination}

To get information about the dynamics of the pellet triggered ELMs, the evolution of their MHD activity was monitored by a set of magnetic pick-up coils located about $10 \mathrm{~cm}$ from the separatrix in the scrape off layer of ASDEX Upgrade [21] (represented by red color on figure 4). It consists of 14 printed circuit coils measuring the variation of the radial magnetic field component. As can be seen on figure 4 the coils are located on the low field side of the vessel, covering a poloidal angle of approximately $60^{\circ}$ (centered on the outer equatorial line; 7 coils - called poloidal pick-up coil set: B31-02, B31-05,.., B31-10) and located toroidally about $220^{\circ}$ from the poloidal cross section of the pellet injection and a toroidal angle of approximately $180^{\circ}$ near to the equatorial plane (5 coils - called toroidal pick-up coil set: B31-02, B31-03, B31-12,.., B31-14). They are particularly suited for the study of the high frequency MHD phenomena related to the ELM cycle, where short timescales are expected as well as high poloidal mode numbers.

Additionally a set of Mirnov coils installed on the inner wall of the AUG vacuum vessel covering a complete poloidal 'circle' (30 coils, represented by green color on figure 4: C09-01,...,C09-32) was also used to detect the ELM onset [22]. These coils are located about $190^{\circ}$ toroidally from the pellet injection.

Analyzing the signals of the magnetic pick-up coils, it was observed that at the very beginning of the ELM event (either a natural or a triggered one) a strong quasi periodic oscillation can be seen on all channels. For the applied plasma scenario this oscillation fell into a frequency range of $100-300 \mathrm{kHz}$. To get the time evolution of the oscillation amplitude, the Hilbert-Huang spectrogram of the signal was calculated for every magnetic coil signal [23]. The advantage of the use of the Hilbert-Huang spectrogram is that this transformation provides the amplitude of any oscillation without significant smoothing which would deteriorate the time resolution. In order to eliminate other non ELM related phenomena, the spectrogram was integrated in the above frequency range and the result is considered as the magnitude of the ELM related MHD 
activity (see one example on figure 1). The onset $\left(t_{E L M}^{B 31-08}\right.$ on figure 1) was defined for every triggered ELM as the time when this magnitude exceeds a predefined appropriate threshold (a fraction of the maximum of an averaged ELM).

We want to determine the onset of this ELM related MHD activity, therefore we have to analyze on which coil signal shows up this activity first. For this purpose the onset time was calculated for every pellet triggered ELM and for every coil. It was observed that most of the onset times calculated from the poloidal pick-up coil set signals differ only with about $\pm 10 \mu s$ for every triggered ELM and for every analyzed shot. Therefore the poloidal pick-up coil set was used to define the ELM onset time for each ELM by averaging the onset times calculated for these coils: $t_{E L M}=\sum_{\text {coil }} t_{E L M}^{c o i l} / N_{\text {coil }}$, where coil index runs for B31-02, B31-05, ... , B31-10. This averaging was made to reduce the error of ELM onset time introduced by the evaluation algorithm. On the other hand the use of the LFS pick-up coils, which are optimized to detect ballooning modes, looks natural because people used to believe that at least the onset of the ELMs is linked to the interaction of the peeling and ballooning modes. The time difference between the onset times and this ELM onset time $\left(\Delta t=t_{E L M}^{c o i l}-t_{E L M}\right)$ for every triggered ELM and coil can be seen on figure 5 for shot $20040\left(v_{P}=240 \mathrm{~m} / \mathrm{s}\right)$ as a function of the coil position. Figure 5 (a), (b) and (c) contain the data obtained for the poloidal pick-up coil set, for the toroidal pick-up coil set and for the Mirnov coils, respectively. The blue circles stand for the individual pellet triggered ELMs. We think that the scattering of the onset times for the individual ELMs seen on this figure is mainly the consequence of the evaluation algorithm. The raw signals carry not only the ELM related informations but possibly other MHD related ones. To reveal the systematic variation of the onset time on the coil location the values averaged over all triggered ELMs are also plotted on this figure (red diamonds). It is obvious that averaged values are in the $[-10,10] \mu \mathrm{s}$ interval around the ELM onset time for every coil positions. Therefore, if there is any systematic variation of the onset time on the coil location it is smaller than $\pm 10 \mu s$ which justifies the further use of the above definition of the ELM onset time with a $\pm 10 \mu \mathrm{s}$ error.

This observation can be quantitatively explained by the fast shear Alfvén wave communication along magnetic field lines, transmitting the magnetic seed perturbation of a developing ELM, wherever started, all around the torus. For instance, taking an Alfvén velocity of $5 \cdot 10^{6} \mathrm{~m} / \mathrm{s}$ and a safety factor $q \simeq 5$ somewhere in the edge pedestal, the magnetic perturbation travels five times around the torus in $10 \mu s$ and at the same time once around the plasma in poloidal direction. Taking into account the possible radial extension of the perturbation and the radial magnetic shear, the magnetic wake has closely passed all magnetic coils during this short time interval.

The situation is not always as simple: it seems to be that for faster pellets $\left(v_{P} \geq 600 \mathrm{~m} / \mathrm{s}\right)$ the onset time can be detected significantly earlier on the Mirnov coils located on the HFS close to the equatorial plane for poloidal angle between $150^{\circ}$ and $210^{\circ}$ (see figure 6 ) than the defined ELM onset time. Having a closer look for the raw signals it seems that an oscillation and/or changes can already be seen a few tens of 
a microsecond before the ELM onset time, but after the time when the pellet crossed the separatrix. The origin of this observation is not yet clear. This perturbation can be caused by the high beta pellet cloud itself moving across the magnetic field with the pellet velocity. The magnetic coils can detect this magnetic perturbation in addition to the magnetic footprint of the developing ELM. Note that the magnetic coils measure the time derivative of the magnetic field, which is probably larger for faster pellets. This speculation is also supported by field line tracing which reveals that the flux bundles starting at the pellet trajectory remain more compact on the HFS and arrive after a half toroidal turn close to the Mirnov coils located at about $150^{\circ}$ and after one and a half toroidal turn close to the Mirnov coils located at about $210^{\circ}$. Following the magnetic field lines on the other direction toroidally (towards LFS) the flux bundles are more diverse and arrive relatively far away from the coils located on the LFS, therefore they can not detect this perturbation. On the other hand the same analysis performed for the naturally occurring ELMs of this discharge does not show this behavior: no systematic variation of the onset time on the coil location was found. This supports that the above observation is caused by the pellet cloud itself. This observation also justifies the usage of the LFS located poloidal pick-up coil set for determining the ELM onset time because these coils seems to be insensitive for the perturbation caused by the pellet cloud itself.

\section{Results and discussion}

As a consequence of the low pellet injection frequency trigger events occur at different times $\left(d t_{\text {elapsed }}\right)$ in the ELM cycle. The dependence of ELM onset delay ( $\left.d t_{E L M_{-} O N S E T}\right)$ - the delay between the ELM onset and the time when the pellet crossed the separatrix - and the MHD energy drop of the ELM collapse on the elapsed time is investigated and the result of the analysis can be seen on figure 7 for two different pellet velocities (right figures for $v_{P}=240 \mathrm{~m} / \mathrm{s}$, left figures for $v_{P}=600 \mathrm{~m} / \mathrm{s}$ ). Pellets could trigger ELMs at any time in the ELM cycle, that is the plasma edge was never stable against a pellet induced seed perturbation: ELMs could be triggered a few millisecond after the onset of a natural ELM which can imply for a few hundred $\mathrm{Hz}$ 'instantaneous' ELM frequency $\left(1 / d t_{\text {elapsed }}\right)$. With increasing elapsed time the ELM onset delay decreases/tends to certain value, and it can be considered to be constant if the elapsed time is larger than $8 \mathrm{~ms}$. The longer delay times for shorter elapsed times is probably due to the fact that the plasma had not enough time to recover after the previous natural ELM collapse. As it is expected the ELM onset delay is shorter for higher velocity probably because the time of flight is shorter.

The relative energy loss seems to be constant for every elapsed time and is slightly smaller than that of the natural one. The slightly smaller energy loss might be explained by the increase of the pedestal collisionality caused by the pellet ablation and mass deposition which decreases/increases the pedestal electron temperature/density. Analysis of the ELM energy losses in type-I ELMy H-modes from several divertor tokamaks showed the relative ELM energy drop with increasing collisionality [24]. 
Here the instantaneous ELM frequency varies at least an order of magnitude therefore this observation indicates that the inverse proportionality between the ELM frequency and the energy expelled during an ELM is not valid for our perturbative ELM triggering approach. One possible explanation for this observation is that the plasma recovers its original parameters - which determines the energy loss during an ELM after a natural ELM on the millisecond timescale. Therefore the energy loss of the perturbative triggered ELM does not depend on the instantaneous ELM frequency.

\subsection{Determination of the trigger location}

According to our preliminary analysis we suppose that to trigger an ELM a pellet has to reach a certain magnetic surface of the plasma (location of the seed perturbation along the pellet trajectory, $l_{\text {seed }}$ ) inside the separatrix independently of its mass and velocity. After the pellet reached this surface the perturbation introduced by the ablating pellet spreads until the trigger position and finally an ELM is initiated, therefore the ELM instability starts to grow. When it reaches the detection threshold of the magnetic pick up coils the ELM onset $\left(t_{E L M}\right)$ is detected. As a consequence the onset of the triggered ELM $\left(t_{E L M}\right)$ is delayed after the pellet entered into the confined plasma $\left(t_{s e p}\right)$ and this ELM onset delay $\left(d t_{E L M}\right.$ ONSET $)$ has two components. One is a time of flight of the pellet from the separatrix $\left(l_{s e p}\right)$ until the location of the seed perturbation and the other - assumed to be pellet velocity independent - is an intrinsic delay $\left(d t_{i n}\right)$ which incorporates the perturbation spread time and the instability growth time:

$$
d t_{E L M \_O N S E T}=t_{E L M}-t_{s e p}=\frac{1}{v_{P}} \cdot\left(l_{\text {seed }}-l_{\text {sep }}\right)+d t_{i n},
$$

where $l_{\text {seed }}$ and $l_{\text {sep }}$ are measured along the pellet trajectory. It is worth noting that the time of flight is measured from the separatrix, because this surface is fixed to the plasma and the geometry of this magnetic surface is usually well documented.

Consistently the measurement of the ELM onset delay as a function of the pellet velocity allows us to determine the location of the seed perturbation and the intrinsic delay time. The results of these measurements can be seen on figure 8 where the ELM onset delay times (elapsed time is larger than $8 \mathrm{~ms}$ ) are plotted against the inverse pellet velocity. The vertical lines on this figure stand for the estimated error of the ELM onset delay of the individual pellet triggered ELMs containing the inaccuracy of the determination of the separatrix coordinates on the HFS, the inaccuracy of the spatial calibration of the camera images, the estimated inaccuracy of the used trajectory reconstruction method (see section 2.2) and the uncertainty of the determination of the ELM onset time $( \pm 10 \mu s)$. It can be stated that the data set does not contradict our assumption therefore a linear function is fitted onto it and beside the time of flight part a clear $50 \pm 7 \mu \mathrm{s}$ offset - intrinsic delay time - is obtained. This indicates that for the first detection of an evolving triggered ELM a delay of $50 \mu \mathrm{s}$ is necessary after the seed perturbation introduced by the ablating pellet reaching the according magnetic surface. 
From the slope of the fitted linear function the location of the seed perturbation can be calculated. It was found to be $2.7( \pm 0.4) \mathrm{cm}$ from the separatrix along the pellet trajectory. In figure 9 the $P(l)=\exp \left(-\left(l-l_{\text {seed }}\right)^{2} / 2 \sigma^{2}\right)\left(l_{\text {seed }}=2.7 \mathrm{~cm}\right.$ and $\left.\sigma=0.4 \mathrm{~cm}\right)$ probability density function of the seed perturbation location is plotted. As well, a typical plasma pressure profile as seen by the pellet along its designated injection path (distance $(l)$ measured from the separatrix) is displayed. The seed location lies with $99 \%$ probability in a narrow range extending $\pm 1.2 \mathrm{~cm}$ around the most probable position in the middle of the pedestal (pedestal top at about $l=6 \mathrm{~cm}$ ). This also indicates HFS injected pellets cannot trigger ELMs in the scrape of layer. Furthermore, a minor part of the technically available pellet mass is ablated yet at ASDEX Upgrade when the seed perturbation is already initiated.

In the present discussion the location of the pellet at the ELM triggering was referred as the position of the seed perturbation. However, due to the gradient of the magnetic field the pellet cloud leaves the pellet from time to time (with the periodicity of 15-20 $\mu \mathrm{s}$ at ASDEX Upgrade) drifting deep into the plasma (from the HFS to the LFS) [25]. The question is whether the cloud attached to the pellet or the detached drifting cloud - which overcomes the pellet - causes the seed perturbation of the ELM triggering. If the latter one is the determining factor then the location of the seed perturbation can be much deeper in the plasma than we concluded previously. Although we have not performed detailed model calculations we think that our assumption is more realistic: the ELM is triggered by the ablating pellet-attached cloud conglomerate. This is supported by the following two arguments.

It was reported [25] that the drifting cloud travels radially about $10 \mathrm{~cm}$ in $10 \mu \mathrm{s}$ in ASDEX Upgrade plasmas, which implies a drift velocity at least 10 times larger than the largest pellet velocity. If this drift velocity determines the location of the seed perturbation then the at least one order of magnitude smaller pellet velocity would not play a role in the time of flight, that is, the ELM onset delay would not depend on the pellet velocity but on the drift velocity which is always the same for the investigated injection scenarios. On the other hand the energy absorbed by the pellet cloud on a magnetic flux surface is proportional to the residence time that is inversely proportional to the cloud velocity. Therefore assuming the same energy absorption for both the detached and attached case - which is an overestimate for the detached cloud - the cooling effect of the drifting cloud is at least one order of magnitude less than for the pellet-attached cloud conglomerate.

\subsection{The effect of the pellet mass on the ELM triggering}

It was shown in the previous section that the ELM onset delay depends on the pellet velocity and on the time elapsed after the previous natural ELM (near to the previous natural ELM). It was assumed by implication that it is independent of the pellet mass. Our investigations confirm this assumption. The mass of the pellets reaching the plasma is not completely predetermined by the centrifuge and the HFS looping system but it has 
a 'natural' variation as well: pellets are sometimes broken or the ice quality is degraded resulting in reduced pellet size/mass than the expected 'ideal' one. This way in one plasma discharge pellets with identical velocity but with different mass - the predefined mass could be randomly reduced by a factor up to 10 - could be identified. The actual relative pellet mass was estimated by the integral value of the ablation monitor signal. This allowed us to investigate how the ELM onset delay depends on the pellet mass. To rule out the $d t_{\text {elapsed }}$ dependence of the ELM onset delay, ELMs at $d t_{\text {elapsed }}>8 \mathrm{~ms}$ were selected. These investigations revealed that the ELM onset delay does not depend on the pellet mass. However, the ablating pellets are not only triggering ELMs but also fueling the plasma and the major part of the pellet mass (more than 80\%) is ablated after the onset of the ELM. This implies that the long term answer of the target plasma - the energy drop caused by the ELM collapse - should be influenced by the pellet mass. To investigate this effect, the size of the triggered ELM - the energy drop of the triggered ELM relative to the MHD energy of the plasma - and the relative size of the triggered ELM - the energy drop of the triggered ELM relative to that of the natural one - were investigated as a function of the pellet mass for the four injection scenarios. As it can be seen of figure 10, where the relative energy drop is plotted versus the particle content of the pellet, a typical triggered ELM is a bit smaller than the previous natural one and the relative energy drop slightly decreases with the pellet size indicating that more fueling reduces the size of the triggered ELMs. The same dependence was obtained for the relative size of the triggered ELM. This behavior might be explained again by the pellet ablation increased pedestal collisionality. Due to technical reasons the larger the pellet mass the smaller its velocity for the applied pellet injection scenarios. Slower pellets deposit more material into unit distance along the pellet path increasing further the collisionality.

\section{Conclusions and outlook}

The pellet triggering of ELMs was investigated by probing the natural ELM cycle injecting pellets with much lower frequency than the natural ELM frequency. It was found that in the investigated parameter range, every pellet can trigger an ELM independently of its velocity and mass and the time elapsed after the previous natural ELM. It is clear that the minimum pellet mass, which is technically realizable in the ASDEX Upgrade pellet injection system, is not optimized for ELM triggering because the major part of the pellet mass is ablated after triggering the ELM causing the unwanted fueling of the plasma.

Supposing that to trigger an ELM a pellet has to reach a certain magnetic surface of the plasma inside the separatrix independently of its mass and velocity, the most probable location of the seed perturbation was found to be in the middle of the pedestal at the high plasma pressure gradient region. This observation is consistent with model calculations (e.g. peeling-ballooning model) of the ELM predicting for tokamaks with type-I ELMs an instability onset localized to the pedestal steep gradient region $[26,27]$. 
It is still a question whether smaller pellets still reaching the same location of the pedestal gradient region can trigger an ELM or not, because the according perturbation reduces with pellet size. To visualize the effect of the pellet mass reduction on the pellet penetration depth and ablation rate the ablation of pellets with four different mass $\left(r_{p}\right.$ (particle content): $0.67 \mathrm{~mm}\left(7 \cdot 10^{19}\right), 0.42 \mathrm{~mm}\left(1.8 \cdot 10^{19}\right), 0.27 \mathrm{~mm}\left(4.3 \cdot 10^{18}\right), 0.17 \mathrm{~mm}$ $\left.\left(1 \cdot 10^{18}\right)\right)$ was simulated by using a neutral gas shielding model [17]. As can be seen on Fig.11 the pellet mass had to be reduced by almost two orders magnitude $(1 / 64)$ to get the pellet completely ablated in the pedestal. In this case the ablation rate and accordingly the particle deposition is reduced by one order of magnitude.

The onset of the MHD signature of the ELMs observed by magnetic coils was detected $50 \pm 7 \mu s$ after the pellet reached the seed position. The question that naturally emerges what is the origin of the observed $50 \mu \mathrm{s}$ intrinsic delay time which incorporates the perturbation spread time and the instability growth time. In the absence of the full understanding of the trigger mechanism only speculative answer can be given for such question.

The perturbation caused by the ablating pellet is accompanied with a large local particle deposition spreading with ion sound speed $\left(\approx 10^{5} \mathrm{~m} / \mathrm{s}\right)$ and with a local cooling which is homogeneously distributed on the magnetic surface on a few $10 \mu \mathrm{s}$ timescale because the fast electron cooling wave travels along the magnetic field lines with electron thermal speed $\left(\approx 10^{7} \mathrm{~m} / \mathrm{s}\right)$. Additionally, the moving high beta pellet cloud formed around the pellet generates a strong MHD perturbation propagating along the magnetic field lines with Alfvén speed $\left(\approx 5 \cdot 10^{6} \mathrm{~m} / \mathrm{s}\right)$, which also appears on the LFS within $50 \mu \mathrm{s}$.

According to the observations ELMs can be triggered either by the cooling of the pedestal region causing a sudden increase of the pedestal plasma pressure gradient driving the plasma to the unstable region of the ballooning mode [28] or by the strong MHD perturbation triggering an instability developing into an ELM. The $50 \mu s$ delay time is not long enough for spreading of ions deposited on the HFS to the LFS, that is this perturbation cannot trigger an ELM on the LFS but taking into account the large local perturbation on the HFS we can not completely exclude that the ELMs are triggered there locally.

To further clarify/investigate the trigger mechanism and validate our present observations ELMs will be triggered by LFS pellet injection which has more flexible technical capabilities. For this purpose a new pellet injector was developed and will be used in the next experimental campaign at ASDEX Upgrade. The same investigations like that one detailed in this paper are planned to be performed. This will probably unveil that the detected $50 \mu \mathrm{s}$ intrinsic delay is HFS specific or it exists on the LFS as well. Combining an LFS injection scenario nearly tangential to the separatrix with horizontal shifting of the plasma the location of the perturbation can be fine tuned which will allow us to map the location of the seed perturbation. 


\section{References}

[1] ITER Physics Expert Groups 1999 Nucl. Fusion 392137

[2] Zohm H 1996 Plasma Phys. Control. Fusion 38105

[3] Herrmann A et al 2002 Plasma Phys. Control. Fusion 44883

[4] Federici F et al 2003 Plasma Phys. Control. Fusion 451523

[5] Lang P T et al 2005 Nucl. Fusion 45502

[6] Lang $\mathrm{P} \mathrm{T}$ et al $1996 \mathrm{Nucl}$. Fusion 361531

[7] Lang P T et al 2003 Nucl. Fusion 431110

[8] Saibene et al 2000 JET Report JET-R(00)02

[9] Baylor L R et al $2001 \mathrm{~J}$. Nucl. Materials 290-293 398

[10] Lang P T et al 2004 Nucl. Fusion 44665

[11] Baylor L R et al 2006 Bull. Am. Phys. Soc. 51115

[12] Geraud A. 2006 24th Soft Conference, Warsaw P4-H-201

[13] Polevoi A R et al 2003 Nucl. Fusion 431072

[14] Lang P T et al 2006 Plasma Phys. Control. Fusion 48 A141

[15] Lang P T et al 2003 Rev. Sci. Instrum. 743974

[16] Horton L D et al 2005 Nucl. Fusion 45856

[17] Parks P B et al 1978 Phys. Fluids 211735

[18] Kocsis G et al 2004 Rev. Sci. Instrum. 754754

[19] Kálvin S et al 2004 Europhysics Conference Abstracts 28G P-5.150

[20] Mc Carthy P J et al 1999 IPP Report 5/85 Max-Planck-Institut fur Plasmaphysik, Garching, Germany

[21] Bolzonella T et al 2004 Plasma Phys. Contr. Fusion 46 A143

[22] Schittenhelm M et al 1997 Nucl. Fusion 371255

[23] Huang N E et al 1998 Proc. R. Soc. Lond. A 454903

[24] Loarte A et al 2002 Plasma Phys. Contr. Fusion 441815

[25] Müller H W et al 2002 Nucl. Fusion 42301

[26] Leonard A W et al 2006 Plasma Phys. Contr. Fusion 48 A149

[27] Snyder P B et al 2005 Physics of Plasmas 12056115

[28] Wilson H R et al 2006 Plasma Phys. Contr. Fusion 48 A71 
$\# 20043, v_{p}=600 \mathrm{~m} / \mathrm{s}$

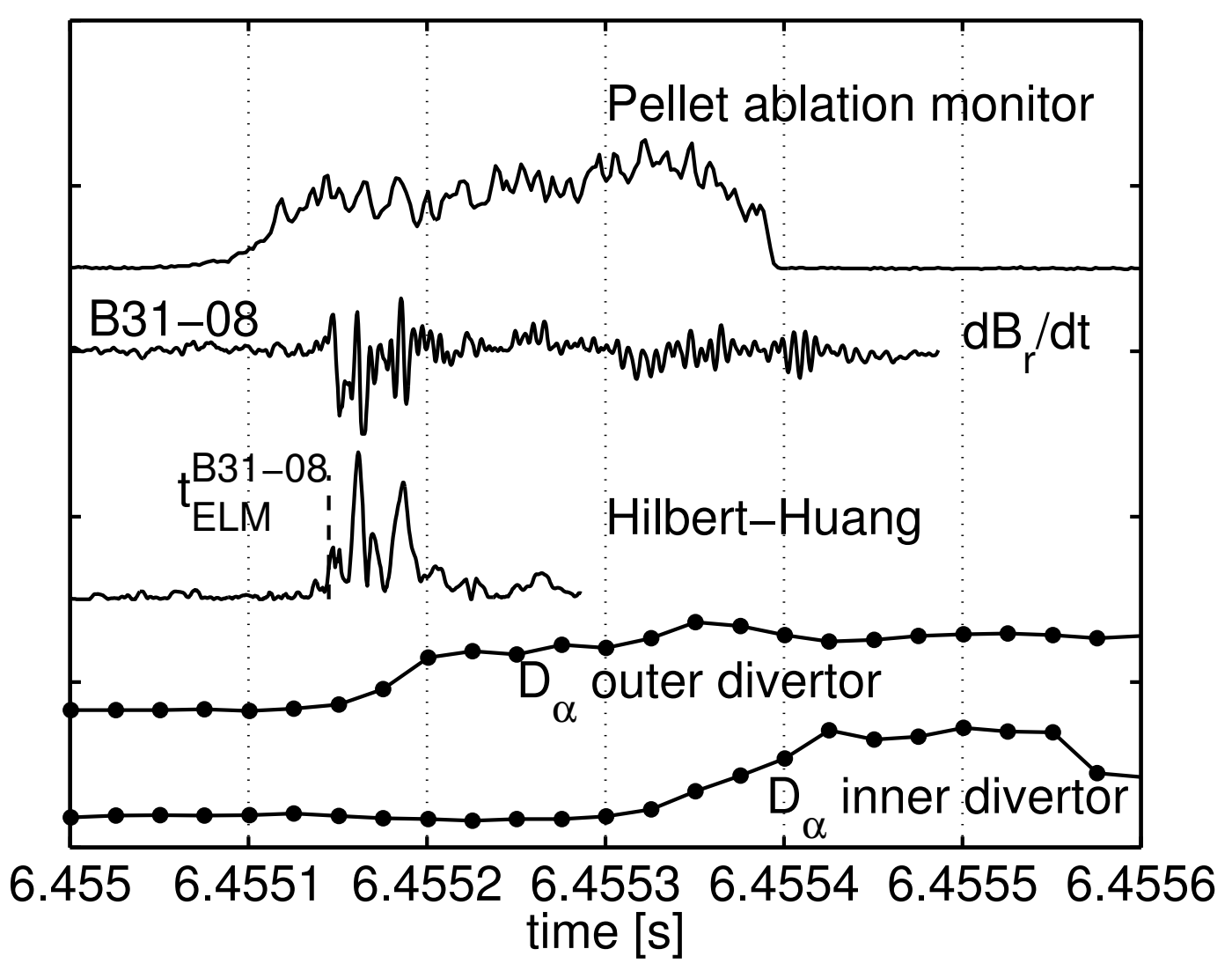

Figure 1. Time traces of ablation monitor signal, magnetic pick-up coil signal $\left(d B_{r} / d t\right)$, Hilbert-Huang spectrogram integrated in the $100-300 \mathrm{kHz}$ frequency range and inner and outer divertor $\mathrm{D}_{\alpha}$ emission during a pellet triggered ELM. 


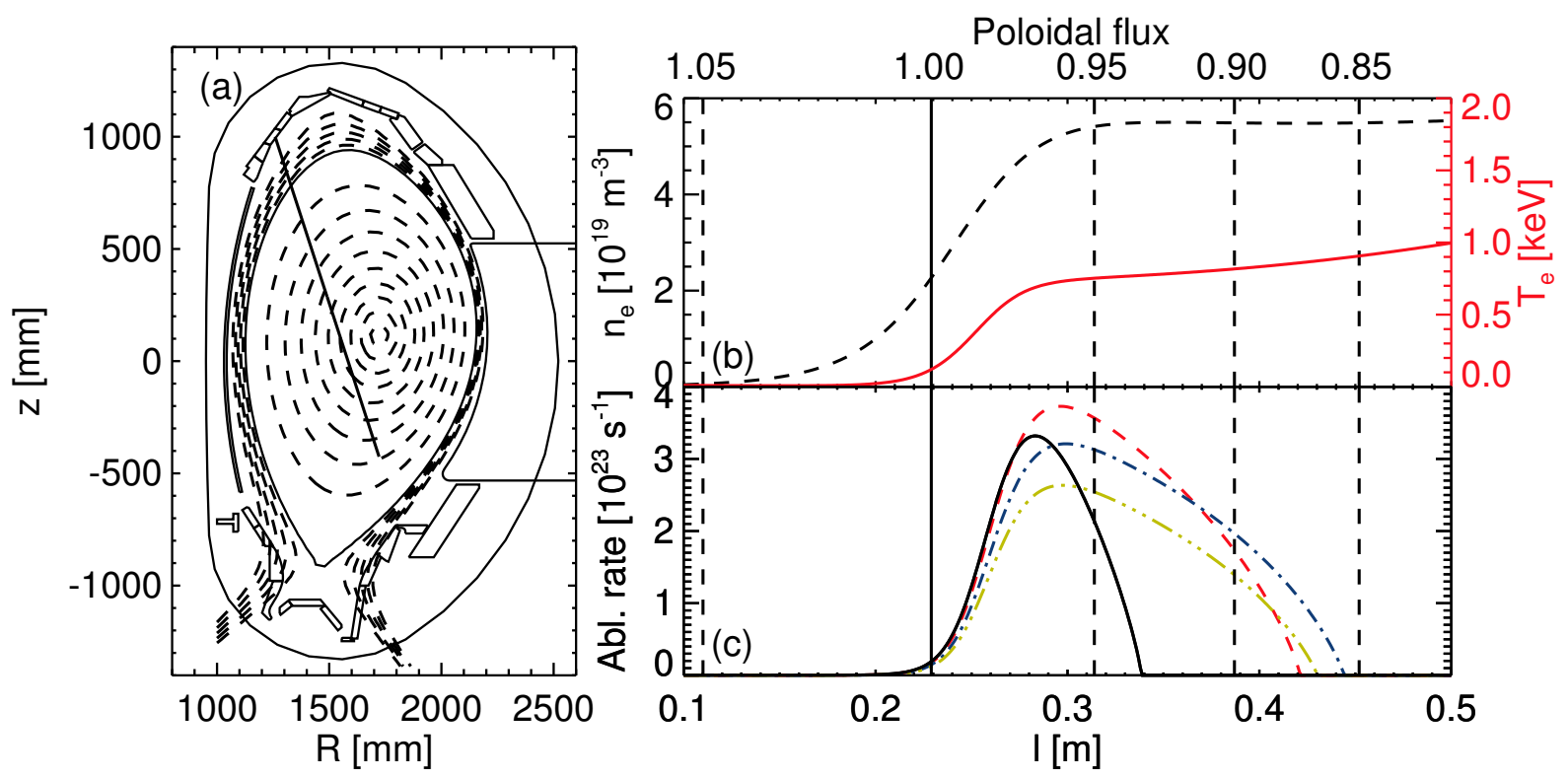

Figure 2. The typical magnetic structure for the investigated discharges (a) together with the designated pellet path (solid line with $72^{\circ}$ declination angle) and typical density (dashed line) and temperature profiles obtained from the combination of different diagnostics and two discharges as a function of the distance along the pellet trajectory (b). The ablation rate calculated by a neutral gas shielding model using these profiles as input data for the four injection scenarios (black solid: $v_{P}=240 \mathrm{~m} / \mathrm{s}$, $r_{P}=0.71 \mathrm{~mm}$, red dashed: $v_{P}=600 \mathrm{~m} / \mathrm{s}, r_{P}=0.67 \mathrm{~mm}$, blue dash dotted: $v_{P}=880 \mathrm{~m} / \mathrm{s}$, $r_{P}=0.58 \mathrm{~mm}$, yellow dash dotted $\left.(-\cdots-): v_{P}=1000 \mathrm{~m} / \mathrm{s}, r_{P}=0.51 \mathrm{~mm}\right)$ as a function of the distance along the pellet trajectory (c). The according normalized poloidal flux coordinates are also shown. 

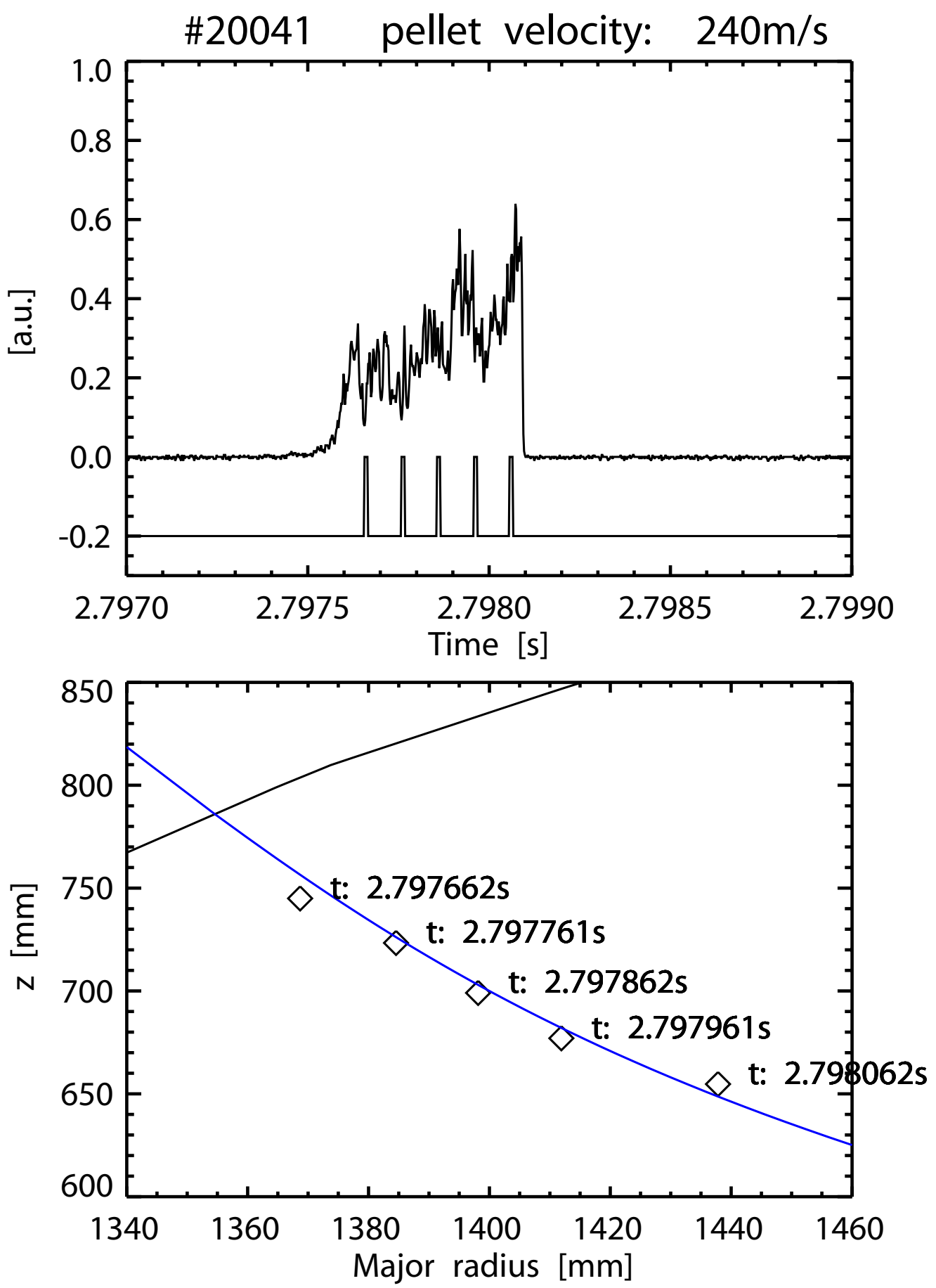

Figure 3. The ablation monitor signal (upper curve) and the camera exposures (lower curve) in a typical discharge for one pellet are shown on the upper figure. The $240 \mathrm{~m} / \mathrm{s}$ velocity pellet has a lifetime of about $600 \mu \mathrm{s}$ allowing us to make five $10 \mu \mathrm{s}$ long exposures in every $100 \mu \mathrm{s}$. On the lower figure the positions of the pellet calculated from the local maxima of the multiple exposure image (diamonds) with the according time stamps and the reconstructed trajectory (blue curve). The black curve represents the separatrix. 

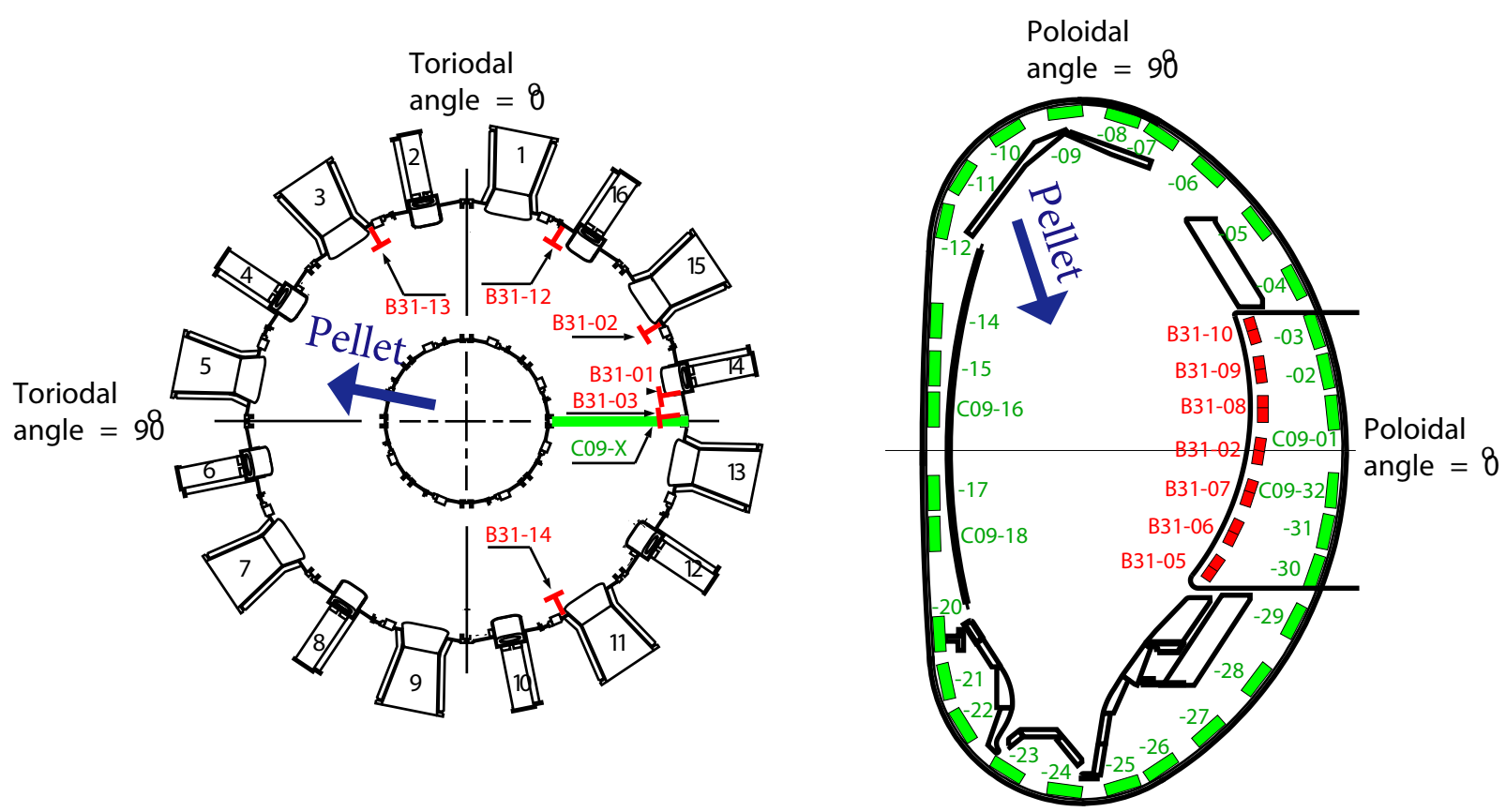

Figure 4. Location of the magnetic pick-up (red) and Mirnov (green) coils. Left figure is a schematic top view, right figure is a combination of two poloidal cross sections (one with the Mirnov coil set and the other with the poloidal pick-up coil set). The location and the direction of pellet injection are also indicated by blue arrows. 


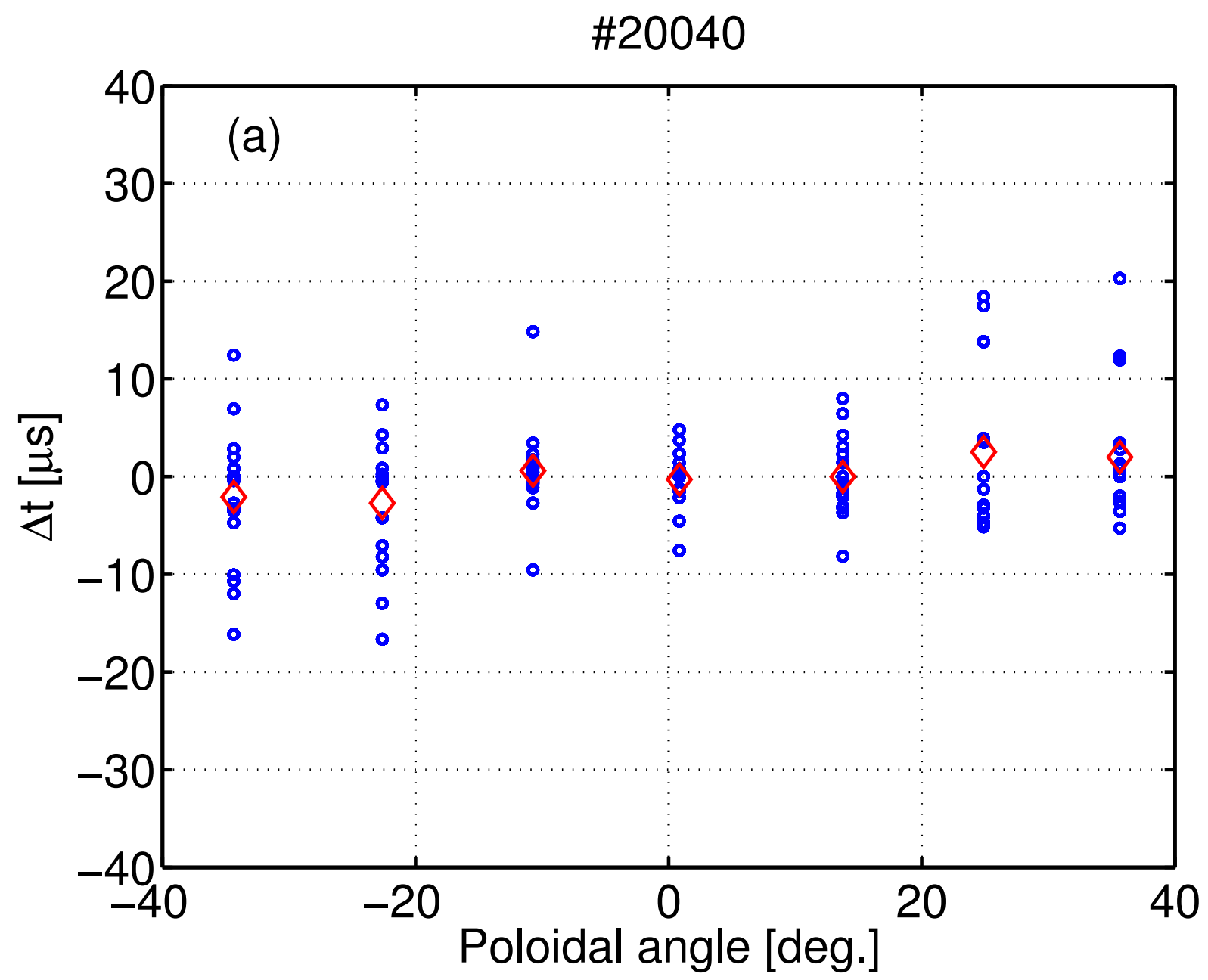

Figure 5. Delay of onset times relative to the ELM onset time (onset times averaged for the poloidal pick-up coil set for each ELM) for the poloidal pick-up coil set (a), for the toroidal pick-up coil set (b) and for the Mirnov coil set (c) for shot 20040 $\left(v_{P}=240 \mathrm{~m} / \mathrm{s}\right)$ as a function of the coil location. The blue circles stand for the individual pellet triggered ELMs and the red diamonds for the values averaged for a given coil. 


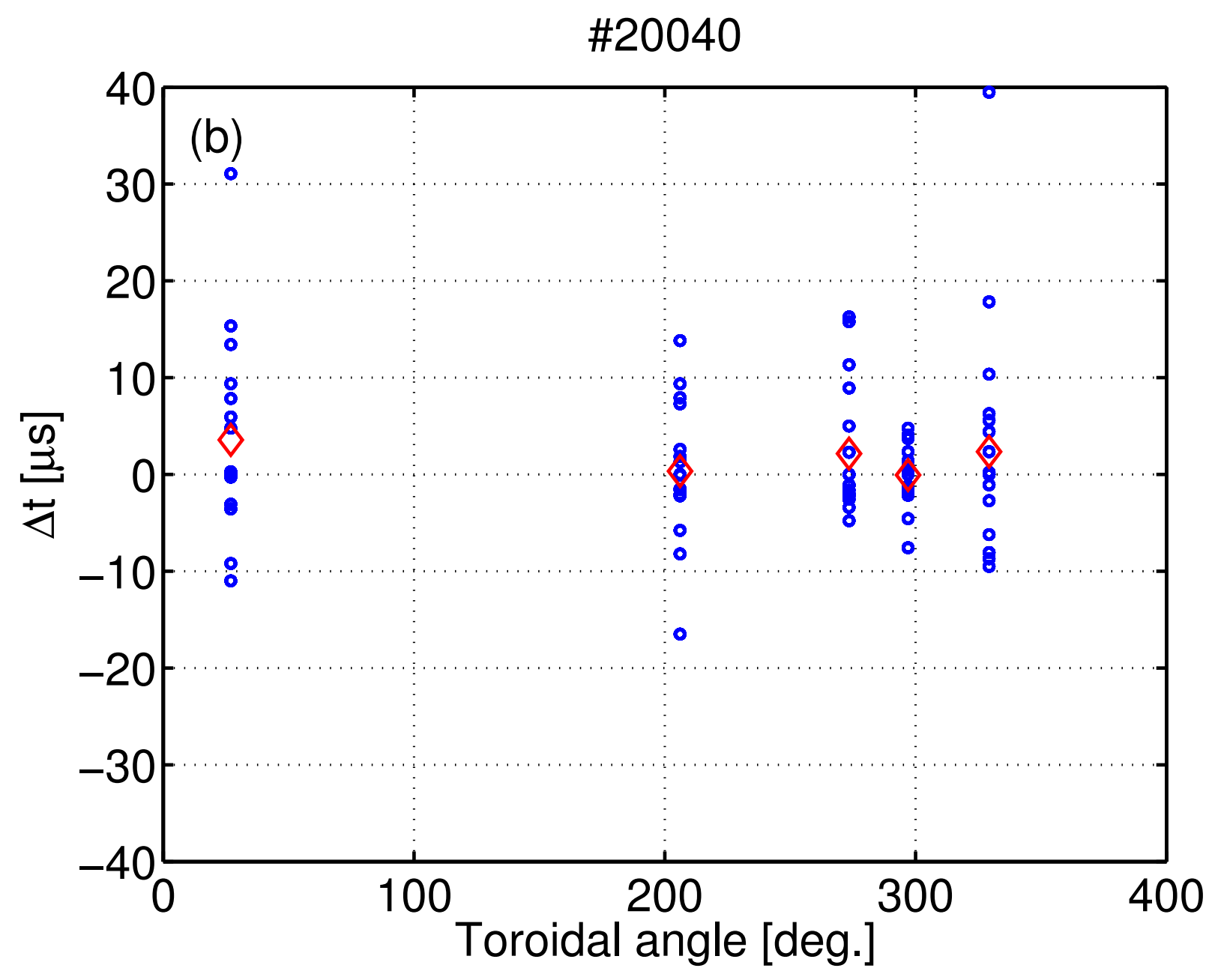




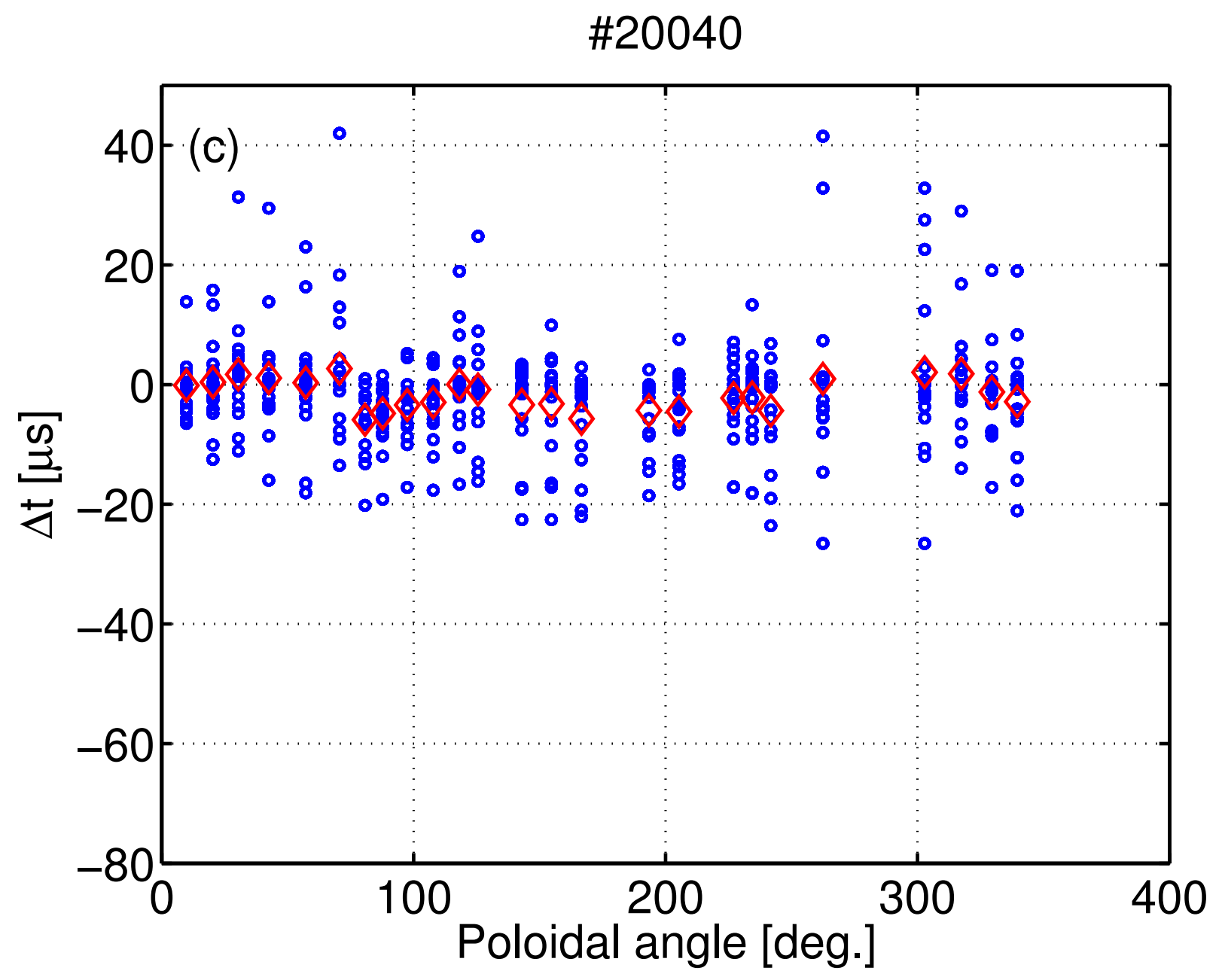




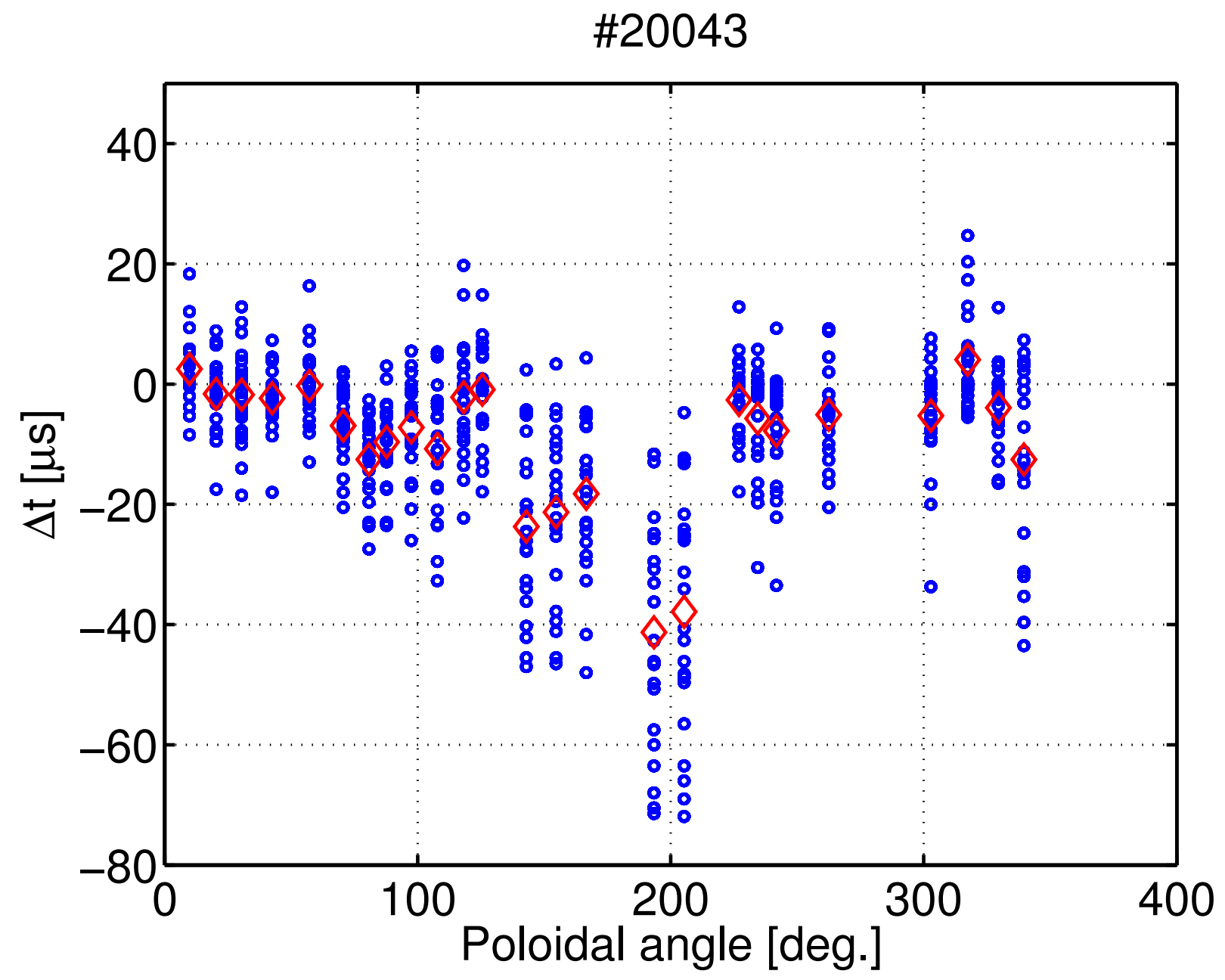

Figure 6. Delay of the onset times relative to the ELM onset time for the Mirnov coil set for shot $20043\left(v_{P}=600 \mathrm{~m} / \mathrm{s}\right)$ as a function of the poloidal angle of the coil location. 

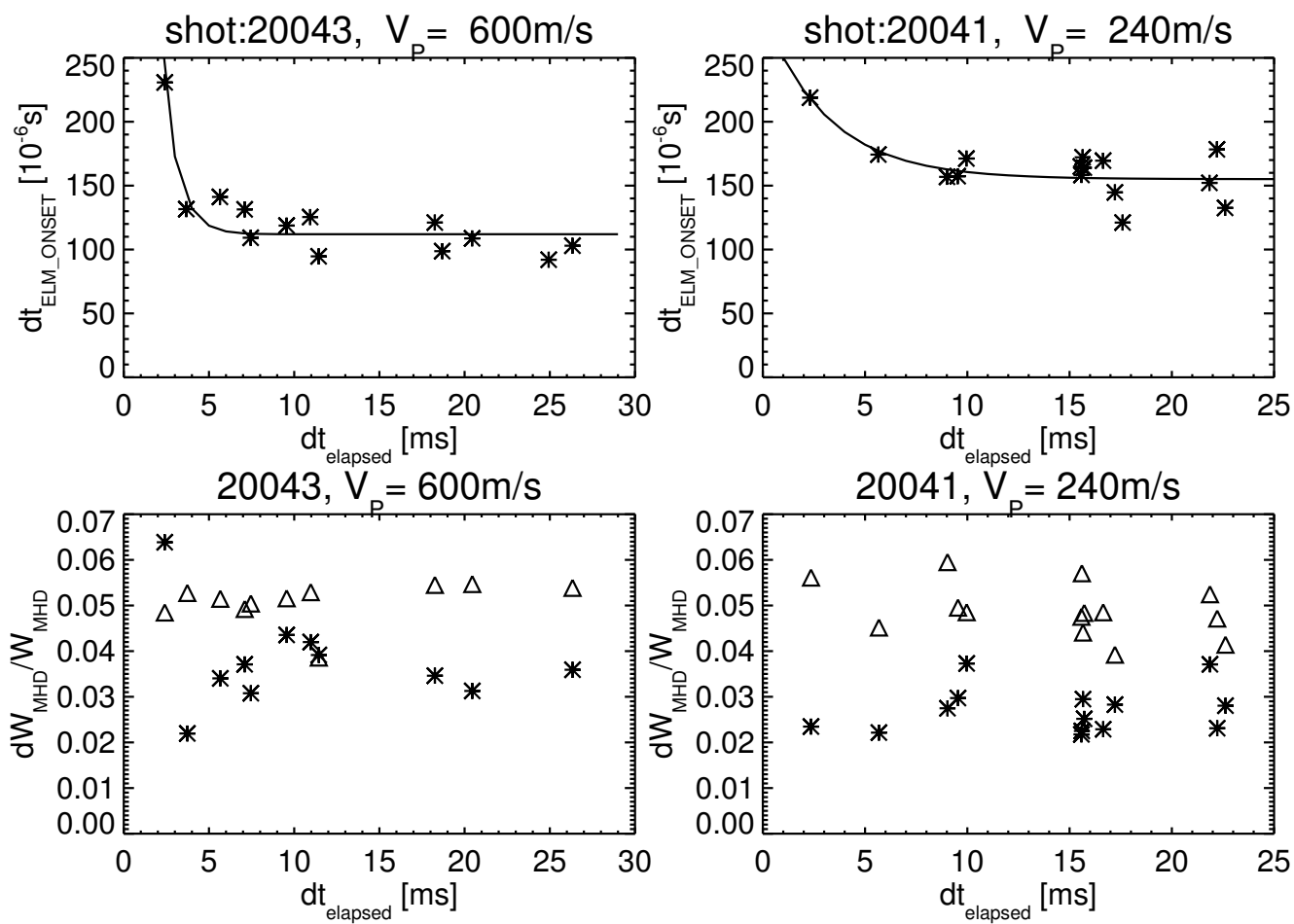

Figure 7. ELM onset delay (upper figures) and relative energy drop of the ELM collapse (lower figures) as a function of elapsed time for two pellet velocities. The fitted curves on the ELM onset delay figure are composed of an offset and exponentially falling function. The stars and the triangles on the lower figures stand for the triggered ELMs and the previous natural ELMs, respectively. 


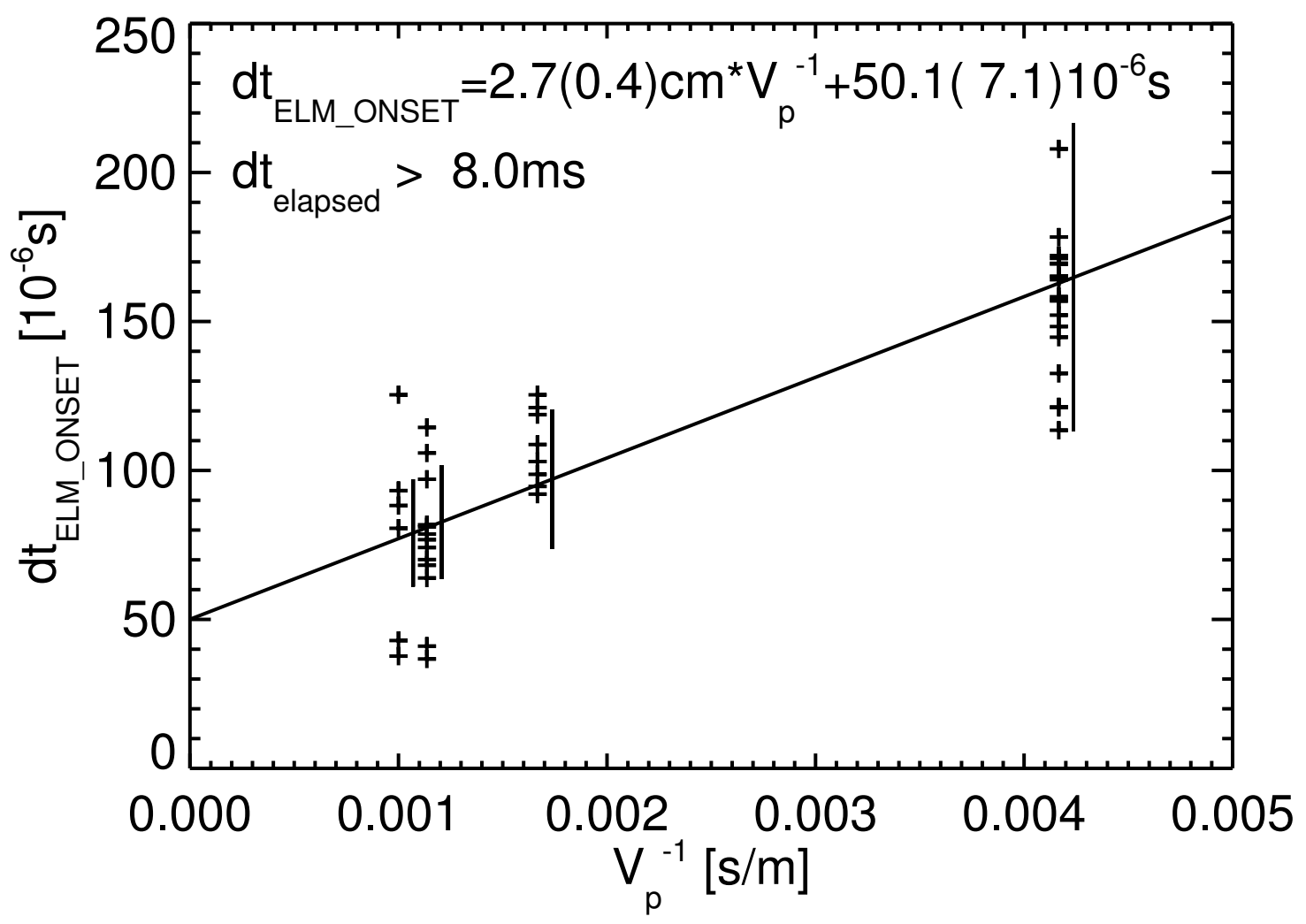

Figure 8. ELM onset delay as a function of the inverse pellet velocity. Vertical lines represent the typical estimated error of the onset delay of the individual ELMs. A fitted linear function is also over plotted. 


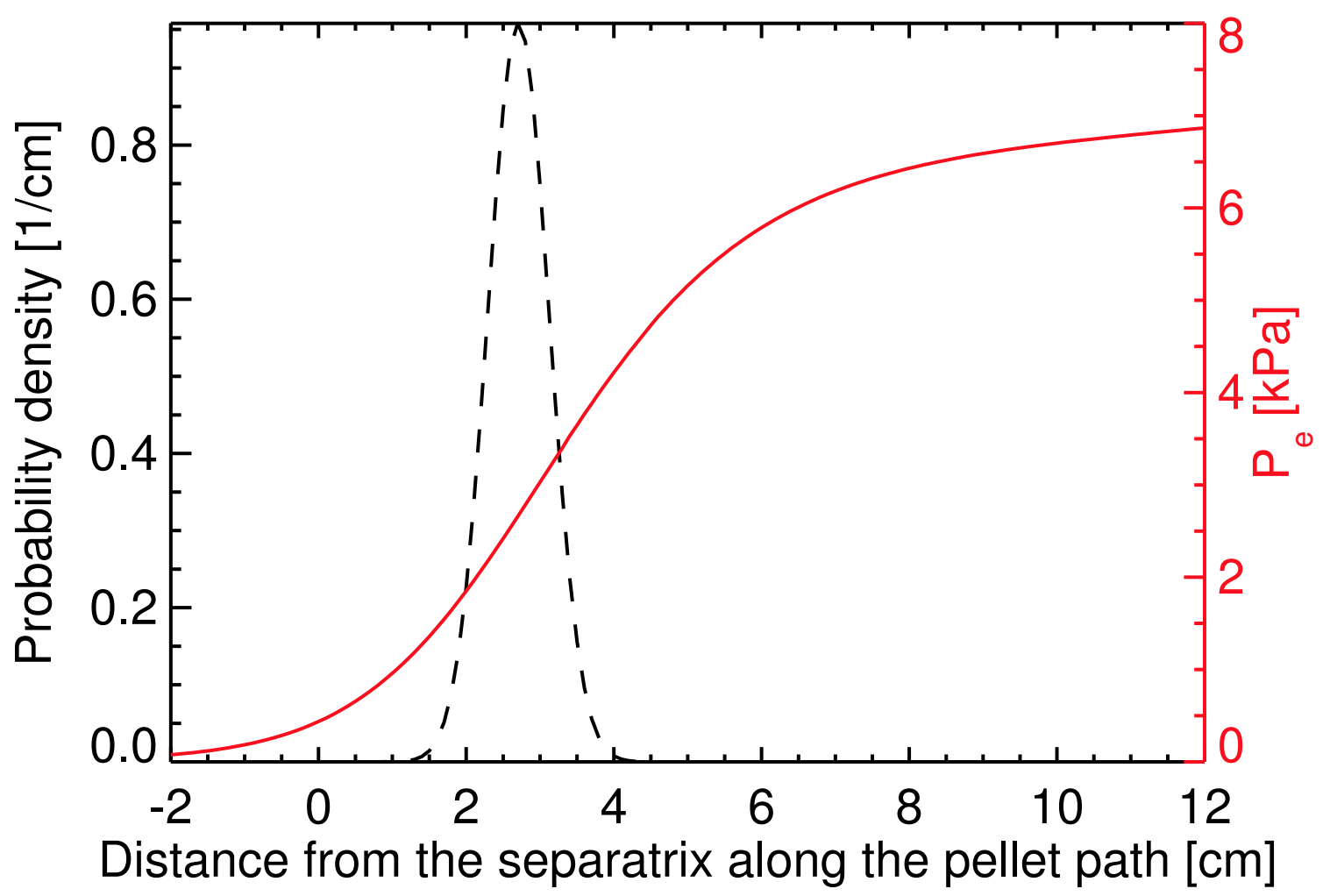

Figure 9. The probability density function of the seed location (dashed curve) and the plasma pressure profile as a function of the distance along the pellet path measured from the position of the separatrix. The pedestal top is at about $6 \mathrm{~cm}$. 


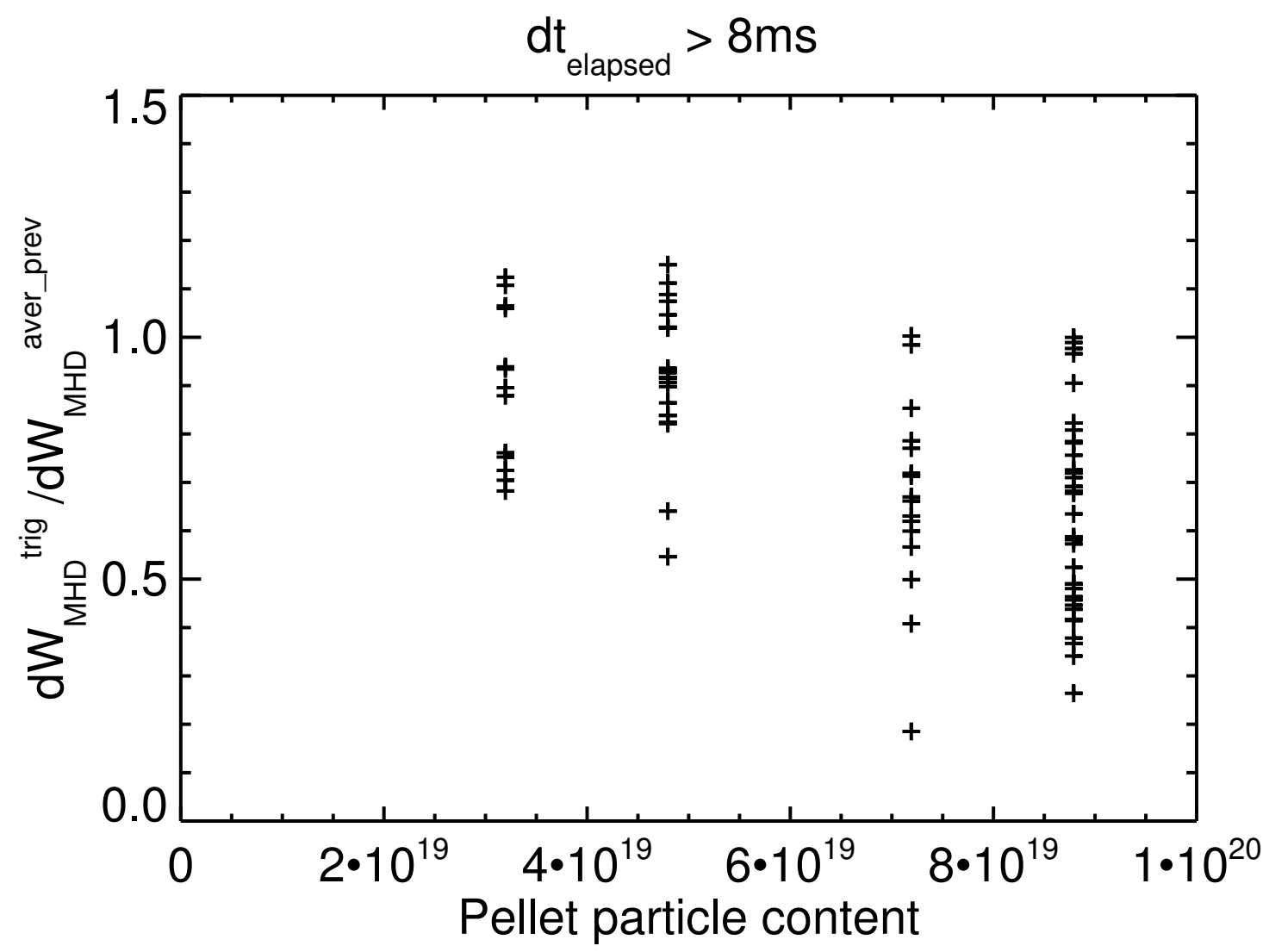

Figure 10. The relative size of the triggered ELM - the energy drop of the triggered ELM relative to that of the natural one - as a function of the expected particle content of the pellets. 


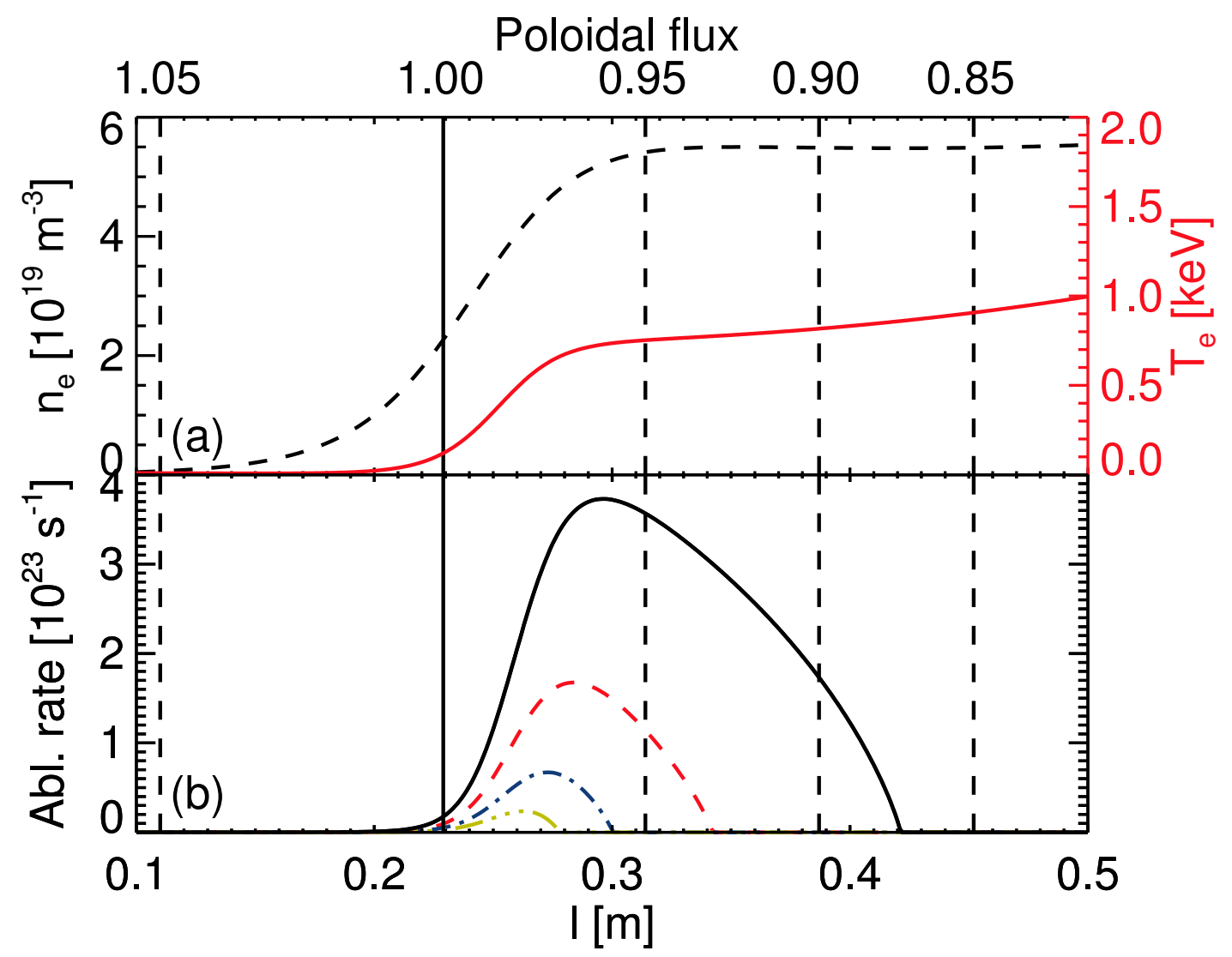

Figure 11. Ablation rate calculated by a neutral gas shielding model for four different pellet sizes (black solid: $r_{P}=0.67 \mathrm{~mm}$, red dashed: $r_{P}=0.42 \mathrm{~mm}$, blue dash dotted: $r_{P}=0.27 \mathrm{~mm}$, yellow dash dotted $\left.(-\cdots-): r_{P}=0.17 \mathrm{~mm}\right)$ as a function of the distance along the pellet trajectory (b). The pellet velocity was set to $v_{P}=600 \mathrm{~m} / \mathrm{s}$ for all four cases. The according normalized poloidal flux coordinates are also shown. On the upper figure (a) the electron density (dashed line) and temperature profiles used in the calculation are seen. 